\title{
ABDOMINAL TUBERCULOSIS MIMICKING OVARIAN CANCER ON CT SCAN: THE VALUE OF CHEST X-RAY IN DIAGNOSIS \\ Ardhi Tripriyanggara $^{\mathrm{a}}$, Lies Mardiyana ${ }^{\mathrm{a}}$, Dyah Erawatia ${ }^{\mathrm{a}}$, Bambang Soeprijanto ${ }^{\mathrm{a}}$, Hari Nugroho ${ }^{\mathrm{b}}$
}

aardhi.t89.at@gmail.com

${ }^{a}$ Department of Radiology, Faculty of Medicine, Airlangga University, Soetomo General Hospital, Surabaya, Indonesia

${ }^{b}$ Department of Obstetrics and Gynaecology, Faculty of Medicine, Airlangga University, Soetomo General Hospital, Surabaya, Indonesia

\begin{abstract}
Background: Difficulties in diagnosing abdominal tuberculosis prevent the patient from receiving optimal therapy. The objective of this study was to determine whether chest X-rays have value in the diagnosis of abdominal tuberculosis which mimics ovarian cancer on CT- scans. Materials and Methods: : This was a retrospective study on abdominal tuberculosis patients who had mimicked ovarian cancer on CT-scans at Soetomo Hospital Surabaya from January 2015 to December 2020. Results: We included 33 patients in this study. The diagnosis was confirmed by biopsy and histopathological examination. The patient age ranged between 20 and 60 years. Twenty patients $(60.6 \%)$ had normal chest X-rays. The most common severity level of CT scans results of abdominal tuberculosis was moderate severity (63.67\%), which was most common in both the group of normal chest X-rays $(65 \%)$ and the group of chest X-rays with pulmonary tuberculosis $(61.5 \%)$. Pearson chi-square test showed there was no statistical relationship between the presence of pulmonary tuberculosis on chest X-ray and the extent of abdominal tuberculosis on CT scan. The contingency coefficient between the variables obtained an $r$ of 0.157 ( $p>0.05$ ). Conclusion: Chest X-ray is considered to have no significant role in the diagnosis of abdominal tuberculosis mimicking ovarian cancer on CT-scan.
\end{abstract}

Published by IJRP.ORG. Selection and/or peer-review under responsibility of International Journal of Research Publications (IJRP.ORG)

Keywords: Abdominal tuberculosis, Chest X-ray, CT scan, Pulmonary tuberculosis

\section{Introduction}

Tuberculosis (TB) is an infection caused by Mycobacterium tuberculosis (MTB) that affects multiorgan system with various presentations and manifestations. Although pulmonary tuberculosis (PTB) is the main form of this disease, extra pulmonary tuberculosis (EPTB) also contributes to TB problems [1]. Of the 6.3 million new TB cases known to WHO in 2017, 16\% were EPTB cases [2]. In 2012, out of 331,424 TB cases, 17,420 EPTB cases were recorded in Indonesia [3]. Abdominal TB incidence ranked sixth in all EPTB cases [4].

Radiology has an important role in diagnosing PTB and abdominal TB. The standard examination used for PTB radiological diagnosis is a plain chest radiograph [5]. In abdominal TB, radiological examinations that could be performed are ultrasound sonography (USG), CT-Scan, and magnetic resonance imaging (MRI) [6].

Generally, the clinical and radiological findings of abdominal TB might be similar to findings in malignancy such as ovarian cancer, or infections such as pelvic inflammatory disease (PID). Some patients with clinical abdominal tumors who were subjected to computed tomography (CT) scans and their findings led to ovarian tumors might be tuberculosis after postoperative biopsy examination is conducted [6-7]. 
Several studies have reported that abdominal TB incidence might coexist with pulmonary TB. The incidence rate varies from $10 \%$ to $50 \%$ depending to the endemic area. In India, the incidents of abdominal TB associated with pulmonary TB was $15 \%$ to $25 \%$ [8]. According to a study conducted in Cameroon in 2010, of all 984 patients, more than a quarter $(26.6 \%)$ had PTB and coexisting abdominal TB [1]. This study aims to determine the correlation between variations in chest X-ray findings of pulmonary tuberculosis and CT scan findings of abdominal TB resembling ovarian masses that have been subjected to histopathological examination.

\section{Materials and Methods}

\subsection{Study Design}

This study was a correlative analytical study with a retrospective approach using coefficient contingency correlation conducted in Dr. Soetomo General Hospital, Surabaya, Indonesia, from January 2015 to December 2020. This study was approved by the local Medical Study Ethics Committee.

\subsection{Study Population and Sample}

The study population was patients with clinical abdominal tumors who were subjected to chest $\mathrm{x}$-ray examination and contrast-enhanced abdominal CT scan. The patient was then performed laparotomy or tumorectomy, followed by a biopsy and a histopathological examination. The study samples were patient's medical records from the study population that met inclusion criteria and was not included in exclusion criteria.

The inclusion criteria for this study were as followed:

- The patient was performed abdominal CT scan and the findings were solid or cystic mass originating from the ovary with or without peritoneal seeding and ascites.

- The patient underwent surgery, and a histopathological examination was performed with the results of TB infection

- The patient was subjected to a chest radiograph

- The presence or absence of TB bacteriological examination results

Exclusion criteria in the study were patients with ovarian tumors who underwent surgery and histopathological examination due to TB but was not performed abdominal CT scans or did not undergo chest radiographs.

\subsection{Data Collection}

Data from abdominal CT scan results in patients with ovarian tumors due to TB infection were re-evaluated by the investigators. The evaluation was conducted by the researcher and a radiologist specializing in female imaging. The evaluation results were summarized in a work sheet. Evaluation of the chest radiograph or the results of an expert's reading (if the x-ray was not found) were conducted. The data were tabulated and analyzed statistically.

The results of the patients' chest radiographs with abdominal TB that resembled ovarian masses were group according to the degree potential infection spread, which were:

- No visible abnormalities on the chest X-ray (normal)

- Inactive PTB (latent PTB) which has less potential infection spread from the lungs to extra pulmonary organs. Chest $\mathrm{X}$-ray findings show fibrosis and calcification.

- Active PTB (primary PTB, miliary PTB, reactive primary post-PTB), which has potential infection spread from the lungs to extra pulmonary organs. Chest $\mathrm{X}$-ray findings show infiltrates or consolidation with or without cavities, pleural effusion, atelectasis, lymphadenopathy, and miliary TB [9].

The degree of abdominal TB extent from abdominal CT-scan findings with contrast was classified based on previous studies and had been published and used the following scoring system:

- Mild, if the score is between 5-8

- Moderate, if the score is between 9-12 
- Severe, if the score is more than 12 [6,10-13].

\subsection{Data Analysis}

Data processing and analysis were conducted using computer software (SPSS ver. 21). Correlation analysis between nominal data and ordinal data were conducted.

\section{Results}

\subsection{Sample Distribution Based on Chest X-Ray Results}

There were 33 samples in this study. Chest $\mathrm{x}$-ray were performed to assess whether any abnormalities were found. If an abnormality was found, it would be classified into active or inactive TB process. Table 1 and 2 show the distribution of chest $\mathrm{x}$-ray findings between samples. Of the 33 samples, there were 20 patients (60.6\%) had no abnormalities in their chest radiograph and 13 patients $(39.4 \%)$ had abnormality in their chest x-rays. Out of 13 patients who had abnormalities on their chest radiograph, there were 12 patients $(92.3 \%)$ with active pulmonary TB and one patient $(7.7 \%)$ with inactive pulmonary TB.

Table 1. Sample distribution based on chest $\mathrm{x}$-ray findings

\begin{tabular}{lcr}
\hline \multicolumn{1}{c}{ Chest Radiograph } & Frequency (n) & Percentage (\%) \\
\hline Normal & 20 & $60.6 \%$ \\
Abnormal & 13 & $39.4 \%$ \\
\hline Total & 33 & $100.00 \%$ \\
\hline
\end{tabular}

Table 2. Sample distribution based on their chest radiograph abnormality type

\begin{tabular}{lrr}
\hline Chest Radiograph & Frequency (n) & Percentage (\%) \\
\hline Active & 12 & $92.3 \%$ \\
Inactive & 1 & $7.7 \%$ \\
\hline Total & 13 & $100.00 \%$ \\
\hline
\end{tabular}

\subsection{Abdominal CT Scan Characteristics}

In abdominal TB patients, there were $7 \mathrm{CT}$ scan findings that were assessed: omental changes, mesenterial changes, peritoneal thickening, lymph nodes enlargement, ascites, ovarian capsule changes, and contrast enhancement in ovarian solid parenchyma. We tried to analyze the differences between CT scan findings of abdominal TB on normal chest radiographs and on chest radiographs for pulmonary TB. There were some patients who did not reveal lesion expansion, which is shown in Table 3.

Table 3. Abdominal TB lesion extension findings on CT scan

\begin{tabular}{lrr}
\hline \multicolumn{1}{c}{ Lesion Characteristics } & Present & \multicolumn{1}{c}{ Not Present } \\
\hline Omental changes & $25(75.7 \%)$ & $8(24.3 \%)$ \\
Mesenterial changes & $28(84.8 \%)$ & $5(15.2 \%)$ \\
Peritoneal thickening & $29(87.8 \%)$ & $4(12.2 \%)$ \\
Lymph node enlargement & $16(48.4 \%)$ & $17(51.6 \%)$ \\
Ascites & $30(90.9 \%)$ & $3(9.1 \%)$ \\
Ovarian capsule changes & $23(69.6 \%)$ & $10(30.4 \%)$ \\
Contrast enhancement & $18(54.5 \%)$ & $15(45.5 \%)$ \\
\hline
\end{tabular}

\subsection{Abdominal CT scan findings on Normal Chest Radiograph}

Table 4 shows the distribution of omental change types in patients with normal chest radiograph. In abdominal TB patients with normal chest radiographs, the most common type was the cake-like type, which 
was found in 13 patients $(65 \%)$. There were three patients $(15 \%)$ who had nodular type, while eight patients (20\%) did not have omental changes.

Table 4. Distribution of omental changes type in abdominal TB patients who had normal chest radiograph

\begin{tabular}{lrr}
\hline \multicolumn{1}{c}{ Omental Changes } & Frequency (n) & Percentage (\%) \\
\hline Cake-like & 13 & $65 \%$ \\
Nodular & 3 & $15 \%$ \\
None & 4 & $20 \%$ \\
\hline Total & 20 & $100 \%$ \\
\hline
\end{tabular}

Table 5 shows the distribution of mesenterial change characteristics in patients with normal chest radiograph, which are: absence of mesenterial changes, nodular infiltration type or mesenterial thickening, and loss of mesenterial configuration. The most common characteristic was nodular infiltration type or mesenterial thickening, which was found in 13 patients $(65 \%)$, then followed by loss of the mesenterial configuration in four patients $(20 \%)$, and absence of mesenterial changes was found in three patients (15\%).

Table 5. Distribution of mesenterial change characteristics in abdominal TB patients who had normal chest radiograph

\begin{tabular}{lrr}
\hline \multicolumn{1}{c}{ Mesenterial Changes } & Frequency (n) & Percentage (\%) \\
\hline Loss of mesenterial configuration & 4 & $20 \%$ \\
$\begin{array}{l}\text { Nodular infiltration type or mesenterial } \\
\text { thickening }\end{array}$ & 13 & $65 \%$ \\
None & 3 & $15 \%$ \\
\hline Total & 20 & $100 \%$ \\
\hline
\end{tabular}

Table 6 shows the distribution of peritoneal thickening types. We divided it into three categories: absence of thickening, thickening with smooth edge, and thickening with lobulated edge. In abdominal TB patients with normal chest radiographs, the most common type was smooth edge which was found in 10 patients $(50 \%)$, then lobulated edge in nine patients (45\%), and absence of thickening was found in only one patient $(5 \%)$.

Table 6. Distribution of peritoneal thickening in abdominal TB patients who had normal chest radiograph

\begin{tabular}{lrr}
\hline \multicolumn{1}{c}{ Peritoneal Thickening } & Frequency (n) & Percentage (\%) \\
\hline Smooth edge & 10 & $50 \%$ \\
Lobulated edge & 9 & $45 \%$ \\
None & 1 & $5 \%$ \\
\hline Total & 20 & $100 \%$ \\
\hline
\end{tabular}

Table 7 the distribution of lymph node enlargement size. These enlargements were divided into two groups: lymph node $>1 \mathrm{~cm}$ and lymph node $<1 \mathrm{~cm}$. In abdominal TB patients with normal chest X-ray, there were equal numbers in each group

Table 7. Distribution of lymph node enlargement size in abdominal TB patients who had normal chest

\begin{tabular}{lrr}
\multicolumn{3}{c}{ radiograph } \\
\hline \multicolumn{1}{c}{ Lymph Node Enlargement } & Frequency (n) & Percentage (\%) \\
\hline$<1 \mathrm{~cm}$ & 10 & $50 \%$ \\
$>1 \mathrm{~cm}$ & 10 & $50 \%$ \\
\hline Total & 20 & $100 \%$ \\
\hline
\end{tabular}


Table 8 shows the presence of ascites in abdominal TB patients who had normal chest radiograph. It turned out that the majority of patients had ascites, which was found in 18 patients (90\%). Two patients (10\%) did not have ascites.

Table 8. Presence of ascites in abdominal TB patients who had normal chest radiograph

\begin{tabular}{lrr}
\hline \multicolumn{1}{c}{ Ascites } & Frequency (n) & \multicolumn{1}{c}{ Percentage (\%) } \\
\hline Present & 18 & $90 \%$ \\
Not present & 2 & $10 \%$ \\
\hline Total & 20 & $100 \%$ \\
\hline
\end{tabular}

Table 9 shows the distribution of ovarian capsule types. There were three types of ovarian capsule: smooth edge, lobulated edge, and nodular edge. In abdominal TB patients with normal chest radiographs, the most common type was lobulated edge in 12 patients (60\%), followed by smooth edge in seven patients (35\%) and nodular edge in one patient $(5 \%)$.

Table 9. Distribution of ovarian capsule types in abdominal TB patients who had normal chest radiograph

\begin{tabular}{lrr}
\hline \multicolumn{1}{c}{ Ovarian Capsule Type } & Frequency (n) & \multicolumn{2}{c}{ Percentage (\%) } \\
\hline Smooth edge & 7 & $35 \%$ \\
Lobulated edge & 12 & $60 \%$ \\
Nodular edge & 1 & $5 \%$ \\
\hline Total & 20 & $100 \%$ \\
\hline
\end{tabular}

Table 10 shows the distribution of contrast enhancement in solid ovarian parenchyma. These enhancements were grouped as followed: $>76 \mathrm{HU}, 40-50 \mathrm{HU}$, and 50-75 HU. Thirteen patients $(65 \%)$ belonged to $>76 \mathrm{HU}$ group, followed by 50-75 HU group, and finally 40-50 HU group.

Table 10. Distribution of contrast enhancement in abdominal TB patients who had normal chest radiograph

\begin{tabular}{|c|c|c|}
\hline $\begin{array}{c}\text { Contrast Enhancement in Solid Ovarian } \\
\text { Parenchyma }\end{array}$ & Frequency $(\mathbf{n})$ & Percentage (\%) \\
\hline$>76 \mathrm{HU}$ & 13 & $65 \%$ \\
\hline $50-75 \mathrm{HU}$ & 6 & $30 \%$ \\
\hline $40-50 \mathrm{HU}$ & 1 & $5 \%$ \\
\hline Total & 20 & $100 \%$ \\
\hline
\end{tabular}

From the above characteristics, we assessed the abdominal TB extension and classified it into 3 degrees. Extension is mild if the value is less than 9, moderate if the value is between 9 to 12 , and severe if the value is more than 12. Table 11 shows that most characteristics fell into moderate degree, which was found in 13 patients $(65 \%)$, mild degree was found in six patients (30\%), and severe degree was only found in one patient $(5 \%)$.

Table 11. Abdominal TB extension degree in normal chest radiograph

\begin{tabular}{lrr}
\hline $\begin{array}{l}\text { Abdominal TB Extension Degree } \\
\text { in Normal Chest Radiograph }\end{array}$ & Frequency (n) & Percentage (\%) \\
\hline Mild & 6 & $30 \%$ \\
Moderate & 13 & $65 \%$ \\
Severe & 1 & $5 \%$ \\
\hline Total & 20 & $100 \%$ \\
\hline
\end{tabular}




\subsection{Abdominal CT Scan Characteristics in Pulmonary TB Chest Radiograph}

Table 12 shows the distribution of omental change types in patients with abnormal chest radiograph. The most common type was the cake-like type, which was found in five patients $(38.4 \%)$. There were four patients $(30.8 \%)$ who had nodular type, while four patients $(30.8 \%)$ did not have omental changes.

Table 12. Distribution of omental changes type in abdominal TB patients who had abnormal chest radiograph

\begin{tabular}{lrr}
\hline \multicolumn{1}{c}{ Omental Changes } & Frequency (n) & Percentage (\%) \\
\hline Cake-like & 5 & $38.4 \%$ \\
Nodular & 4 & $30.8 \%$ \\
None & 4 & $30.8 \%$ \\
\hline Total & 13 & $100.0 \%$ \\
\hline
\end{tabular}

Table 13 shows the distribution of mesenterial change characteristics in patients with abnormal chest radiograph. The most common characteristic was nodular infiltration type or mesenterial thickening, which was found in 10 patients $(77.0 \%)$, while loss of the mesenterial configuration was found in one patient $(7.7 \%)$, and absence of mesenterial changes was found in two patients $(15.3 \%)$.

Table 13. Distribution of mesenterial change characteristics in abdominal TB patients who had abnormal chest radiograph

\begin{tabular}{lrr}
\hline \multicolumn{1}{c}{ Mesenterial Changes } & Frequency (n) & Percentage (\%) \\
\hline Loss of mesenterial configuration & 1 & $7.7 \%$ \\
$\begin{array}{l}\text { Nodular infiltration type or mesenterial } \\
\text { thickening }\end{array}$ & 10 & $77.0 \%$ \\
None & 2 & $15.3 \%$ \\
\hline Total & 13 & $100.0 \%$ \\
\hline
\end{tabular}

Table 14 shows the distribution of peritoneal thickening types. In abdominal TB patients with abnormal chest radiographs, smooth edge was found in five patients $(38.5 \%)$, lobulated edge was also found in five patients, and absence of thickening was found in only three patients $(23.0 \%)$.

Table 14. Distribution of peritoneal thickening in abdominal TB patients who had abnormal chest radiograph

\begin{tabular}{lrr}
\hline \multicolumn{1}{c}{ Peritoneal Thickening } & Frequency $(\mathbf{n})$ & Percentage (\%) \\
\hline Smooth edge & 5 & $38.5 \%$ \\
Lobulated edge & 5 & $38.5 \%$ \\
None & 3 & $23.0 \%$ \\
\hline Total & 20 & $100.0 \%$ \\
\hline
\end{tabular}

Table 15 the distribution of lymph node enlargement size. Most patients had lymph node enlargement of $<1 \mathrm{~cm}$, which was found in seven patients $(53.8 \%)$, and the rest had $>1 \mathrm{~cm}$ of lymph node enlargement $(46.2 \%)$.

Table 15. Distribution of lymph node enlargement size in abdominal TB patients who had abnormal chest

\begin{tabular}{lrr}
\multicolumn{3}{c}{ radiograph } \\
\hline \multicolumn{1}{c}{ Lymph Node Enlargement } & Frequency (n) & Percentage (\%) \\
\hline$<1 \mathrm{~cm}$ & 7 & $53.8 \%$ \\
$>1 \mathrm{~cm}$ & 6 & $46.2 \%$ \\
\hline Total & 13 & $100 \%$ \\
\hline
\end{tabular}


Table 16 shows the presence of ascites in abdominal TB patients who had abnormal chest radiograph. It turned out that the majority of patients had ascites, which was found in 12 patients $(92.3 \%)$. Only one patient (7.7\%) did not have ascites.

Table 16. Presence of ascites in abdominal TB patients who had abnormal chest radiograph

\begin{tabular}{lrr}
\hline \multicolumn{1}{c}{ Ascites } & Frequency (n) & Percentage (\%) \\
\hline Present & 12 & $92.3 \%$ \\
Not present & 1 & $7.7 \%$ \\
\hline Total & 20 & $100.0 \%$ \\
\hline
\end{tabular}

Table 17 shows the distribution of ovarian capsule types. In abdominal TB patients with abnormal chest radiographs, the most common type was lobulated edge in 8 patients $(61.5 \%)$, followed by smooth edge in three patients $(23.2 \%)$ and nodular edge in two patients $(15.3 \%)$.

Table 17. Distribution of ovarian capsule types in abdominal TB patients who had abnormal chest radiograph

\begin{tabular}{lrr}
\hline \multicolumn{1}{c}{ Ovarian Capsule Type } & Frequency (n) & Percentage (\%) \\
\hline Smooth edge & 3 & $23.2 \%$ \\
Lobulated edge & 8 & $61.5 \%$ \\
Nodular edge & 2 & $15.3 \%$ \\
\hline Total & 13 & $100.0 \%$ \\
\hline
\end{tabular}

Table 18 shows the distribution of contrast enhancement in solid ovarian parenchyma. Eight patients (61.5\%) belonged to 50-76 HU group, and the rest belonged to >76 HU group (38.5\%).

Table 18. Distribution of contrast enhancement in abdominal TB patients who had abnormal chest radiograph

\begin{tabular}{|c|c|c|}
\hline $\begin{array}{c}\text { Contrast Enhancement in Solid Ovarian } \\
\text { Parenchyma }\end{array}$ & Frequency (n) & Percentage (\%) \\
\hline$>76 \mathrm{HU}$ & 5 & $61.5 \%$ \\
\hline $50-75 \mathrm{HU}$ & 8 & $38.5 \%$ \\
\hline $40-50 \mathrm{HU}$ & 0 & $0.0 \%$ \\
\hline Total & 20 & $100.0 \%$ \\
\hline
\end{tabular}

From the above characteristics, we assessed the abdominal TB extension and classified it into 3 degrees. Table 19 shows that most characteristics fell into moderate degree, which was found in eight patients $(61.5 \%)$, mild degree was found in five patients $(38.5 \%)$, and no patient had severe degree.

Table 19. Abdominal TB extension degree in abnormal chest radiograph

\begin{tabular}{lrr}
\hline $\begin{array}{c}\text { Abdominal TB Extension Degree } \\
\text { in Abnormal Chest Radiograph }\end{array}$ & Frequency (n) & \multicolumn{2}{c}{ Percentage (\%) } \\
\hline Mild & 5 & $38.5 \%$ \\
Moderate & 8 & $61.5 \%$ \\
Severe & 0 & $0.0 \%$ \\
\hline Total & 13 & $100.0 \%$ \\
\hline
\end{tabular}




\subsection{Distribution of Pulmonary TB Extension on Chest Radiograph with Abdominal TB Extension on Abdominal} CT Scan

Table 20 shows the classification of chest radiograph findings of pulmonary TB with abdominal TB extension on abdominal CT scan. Far advanced lesions on chest radiograph were found in eight patients, followed by minimal lesions (four patients) and chronic lesion (one patient).

Table 20. Distribution of pulmonary TB extension on chest radiograph with abdominal TB extension on

\begin{tabular}{|c|c|c|c|}
\hline $\begin{array}{c}\text { Chest Radiograph } \\
\text { Classification }\end{array}$ & $\mathbf{n}$ & $\begin{array}{c}\text { Abdominal TB Extension on } \\
\text { Abdominal CT Scan }\end{array}$ & $\mathbf{n}$ \\
\hline \multirow[t]{3}{*}{ Minimal lesion } & 4 & Mild & 4 \\
\hline & & Moderate & - \\
\hline & & Severe & - \\
\hline \multirow[t]{3}{*}{ Far advanced lesion } & 8 & Mild & 4 \\
\hline & & Moderate & 4 \\
\hline & & Severe & - \\
\hline \multirow[t]{3}{*}{ Chronic lesion } & 1 & Mild & 1 \\
\hline & & Moderate & - \\
\hline & & Severe & - \\
\hline
\end{tabular}

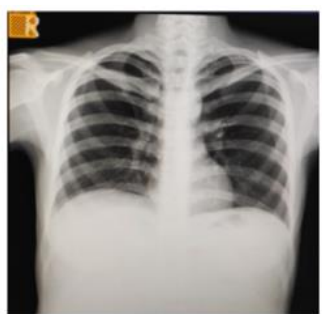

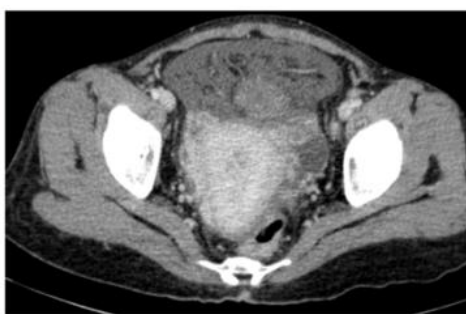

b

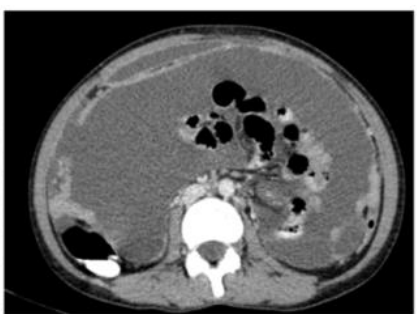

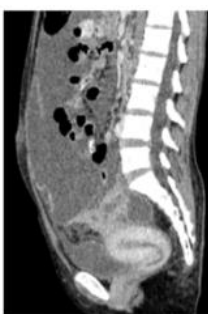

d

Figure 1. (a) A 30-year-old woman with abdominal tuberculosis. The chest X ray was within normal limits. (b) The contrast-enhanced CT scan shows ill-defined, mixed (solid and cystic) left adnexal mass with multilocular caseous necrotic enhancement. (c and d) The axial and sagittal CT image showed a large ascites, and nodulous thickened and enhanced peritoneum. The scoring system based on CT scan findings was classified as a moderate level of severity (score $=8$ ).

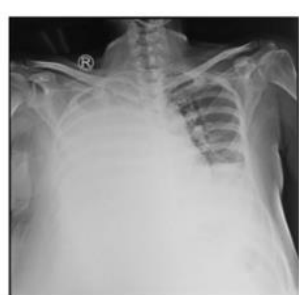

a

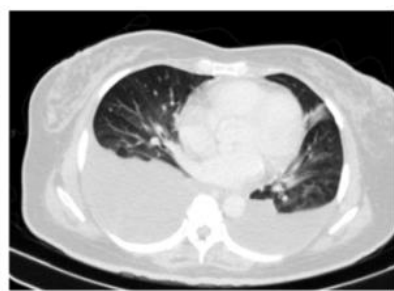

b

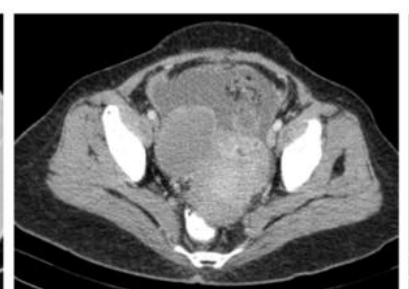

c

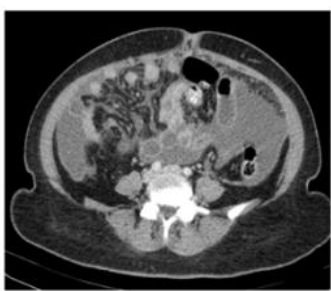

d

Figure 2. A 47-year-old woman with abdominal tuberculosis. (a) The chest $X$ ray showed complete opacification of the right hemithorax consistent with massive pleural effusion. The mediastinum appears shifted to the right side. Fewer pleural effusions at the base of the left hemithorax was also seen. (b) The unenhanced CT scan of the chest revealed bilateral pleural effusion. (c and d) The contrast-enhanced CT scan of the abdomen and pelvis shows an ovoid, ill-defined, mixed (solid and cystic) right adnexal mass, with ascites and multilocular caseous necrotic enhancement. Thickened and enhanced peritoneum was noted. The scoring system based on CT scan findings was classified as a moderate level of severity $($ score $=11)$ 
3.6. Correlation between Variations of Pulmonary TB Chest Radiograph Findings with Variations of Abdominal TB CT Scan Findings

This study used a contingency correlation coefficient. The results of the correlation test were not significant with $p$ value of $0.659(p>\alpha$, with $\alpha=0.05)$ and rho value $=0.157$. The results of the correlation test showed that there was no significant correlation between pulmonary TB chest radiograph findings and CT findings of abdominal TB which resembled ovarian mass.

\section{Discussion}

In this study, 33 samples with abdominal TB that resembled ovarian mass were obtained, which were subjected to a chest x-ray, abdominal CT scan with contrast, surgery, and their biopsy results came out as TB infection. From all those abdominal TB patients, 13 patients $(39.4 \%)$ had pulmonary TB findings on their chest $\mathrm{X}$-rays. In India, the incidence rate of pulmonary TB findings in abdominal TB patients was reported to reach $15 \%$ to $25 \%$ [8], while in China it reached $53.57 \%$ [14].

According to Debi et al, there are various underlying factors that might explain extrapulmonary TB findings: direct infection from infected food or sputum, primary focus on the lungs that spreads through the blood vessels or lymph nodes to digestive tract, and direct infection from surrounding organs such as the fallopian tubes and adnexa to peritoneum [15].

Currently, there is no available classification to determine the degree of abdominal tuberculosis extension that resembles ovarian mass. Therefore, we tried to arrange a scoring system based on previous studies regarding abdominal TB characteristics [6,10-13]. The lowest score was 4, which was included in mild degree category, and the highest score was 13 , which was included in severe degree category. The distribution of the degree of extension were variable in this study, where most of them were included in moderate degree.

We tested the correlation between TB findings on chest X-ray and abdominal TB findings which resembled ovarian mass on abdominal CT scan. In samples with normal chest radiographs, it was found that 13 samples $(65 \%)$ showed a moderate degree of abdominal TB findings that resembled an ovarian mass on abdominal CT scan, six samples $(30 \%)$ had a mild degree, and one sample (5\%) had a severe degree of abdominal TB. Meanwhile, the chest radiograph with pulmonary tuberculosis, whether active or inactive, showed that eight samples $(61.5 \%)$ had moderate degree of abdominal TB and five samples (38.5\%) had mild degree of abdominal TB.

The findings above were then tested with a correlation test using contingency correlation coefficient. The results showed that there was no significant relationship between variations of pulmonary TB chest X-ray findings with variations of abdominal TB findings that resembled ovarian mass on abdominal CT scan. This could be due to the small number of samples; therefore, samples were not evenly distributed and there was a modification to the classification of extension degree of abdominal TB which resembles ovarian mass extension, which need further investigation.

\section{Conclusion}

The chest X-ray findings of pulmonary tuberculosis do not have a significant role in the diagnosis of abdominal tuberculosis mimicking ovarian cancer on CT scans. This is based on the findings in this study, which were as follows:

1. Most of the patients' chest X-rays showed normal features $(60.6 \%)$.

2. The severity of CT scan results of abdominal tuberculosis was mostly moderate in the group with normal chest X-rays (65\%) and in the group with pulmonary tuberculosis on chest X-rays (61.5\%).

3. The Pearson chi-square test analysis showed that the variables was independent of each other and there was no statistical relationship between the presence of pulmonary tuberculosis on chest X-ray and the extent of abdominal tuberculosis on CT scan. 
4. Contingency analysis showed that there was a weak correlation between the presence of pulmonary tuberculosis on chest X-ray and the extent of abdominal tuberculosis on CT scan.

\section{Acknowledgements}

We would like to thank Department of Radiology of Dr. Soetomo Hospital Surabaya, Indonesia in providing the data for analysing

\section{References}

1. Houda, A. and Makram, K. (2018) 'Extrapulmonary Tuberculosis: Update on the Epidemiology, Risk Factors and Prevention Strategies', International Journal of Tropical Diseases, 1(1). doi: 10.23937/ijtd2017/1710006.

2. Pang, Y., An, J., Shu, W., Huo, F., Chu, N., Gao, M., et al. (2019). Epidemiology of extrapulmonary tuberculosis among inpatients, China, 2008-2017. Emerging infectious diseases, 25(3), 457.

3. Suhariani, W. and Wibisono, B. (2015) 'POLA KLINIK TUBERKULOSIS EKSTRA PARU DI RSUP Dr. KARIADI SEMARANG PERIODE JULI 2013- AGUSTUS 2014', Jurnal Kedokteran Diponegoro, 4(4), pp. 1638-1652.

4. Urabinahatti, K. A., Singh, A. K., Nayak, A., Gupta, R., Jain, M., Dubey, C., \& Garg, R. K. (2016). Abdominal tuberculosis: an epidemiological profile and management of 40 cases in a tertiary set up. International Surgery Journal, 3(3), 1502-1508.

5. Nachiappan, A. C., Rahbar, K., Shi, X., Guy, E. S., Mortani Barbosa Jr, E. J., Shroff, G. S., et al. (2017). Pulmonary tuberculosis: role of radiology in diagnosis and management. Radiographics, 37(1), 52-72.

6. Sah, S. K., Shi, X., Du, S., Li, X., Li, C. H., Shah, S., et al. (2017). CT findings and analysis for misdiagnosis of female pelvic tuberculosis. Radiology of Infectious Diseases, 4(1), 19-25.

7. Lalwani, N., Patel, S., Ha, K. Y., Shanbhogue, A. K., Nagar, A. M., Chintapalli, K. N., \& Prasad, S. R. (2012). Miscellaneous tumour-like lesions of the ovary: cross-sectional imaging review. The British journal of radiology, 85(1013), 477-486.

8. Ahuja, V. (2019) 'Abdominal tuberculosis.', Calcutta medical journal, 49(9), pp. 362-366.

9. Agyeman, A. A. and Ofori-Asenso, R. (2017) 'Tuberculosis - an overview', Journal of Public Health and Emergency, 1(3), pp. 7-7. doi: 10.21037/jphe.2016.12.08.

10. Noor, S., Nahar, N., Bilkis, A., \& Jabin, T. (2015). Abdomino Pelvic Tuberculosis Versus Advanced Ovarian Malignancy: A Diagnostic Dilemma. Chattagram Maa-O-Shishu Hospital Medical College Journal, 14(2), 65-69.

11. Hasanzadeh, M., Naderi, H. R., Hoshyar, A. H., Shabane, S., \& Shahidsales, S. (2014). Female genital tract tuberculosis presenting as ovarian cancer. Journal of research in medical sciences: the official journal of Isfahan University of Medical Sciences, 19(2), 184.

12. Fahmi, M. N. and Harti, A. P. (2019) 'A diagnostic approach for differentiating abdominal tuberculosis from ovarian malignancy: A case series and literature review', BMC Proceedings. BMC Proceedings, 13(1), pp. 1-8. doi: 10.1186/s12919-019-0180-y.

13. Shim, S. W., Shin, S. H., Kwon, W. J., Jeong, Y. K., \& Lee, J. H. (2017). CT differentiation of female peritoneal tuberculosis and peritoneal carcinomatosis from normal-sized ovarian cancer. Journal of computer assisted tomography, 41(1), 32-38.

14. Liu, Q., Zhang, Q., Guan, Q., Xu, J. F., \& Shi, Q. L. (2014). Abdominopelvic tuberculosis mimicking advanced ovarian cancer and pelvic inflammatory disease: a series of 28 female cases. Archives of gynecology and obstetrics, 289(3), 623-629.

15. Debi, U., Ravisankar, V., Prasad, K. K., Sinha, S. K., \& Sharma, A. K. (2014). Abdominal tuberculosis of the gastrointestinal tract: revisited. World Journal of Gastroenterology: WJG, 20(40), 14831 